\title{
A novel subdermal anchoring technique for the effective treatment of congenital melanocytic nevus using de-epithelialized dermal flaps
}

\author{
Jin Woo Han, Hook Sun, Jin Woo Kim, Ji Young Yun, Eui Han Chung, Min Jun Oh \\ Department of Plastic and Reconstructive Surgery, Busan Paik Hospital, Inje University School of Medicine, Busan, Korea
}

\begin{abstract}
Background In patients with congenital melanocytic nevus (CMN), single-stage removal of large lesions can be difficult because the high tension created by excising and repairing a large lesion may result in scar widening. Herein, we introduce a method to effectively excise lesions while minimizing scarring and compare its outcomes to those of existing surgical methods.

Methods We compared patients who underwent surgery using the anchoring technique $(n=42)$ or the conventional elliptical technique $(n=36)$. One side of the lesion was removed via en bloc resection up to the superficial fascia. The other side of the lesion was removed via de-epithelialization. The de-epithelialized dermal flap was then fixed by suturing it to the superficial fascia on the opposite side. The length of the lesion's long axis and amount of scar widening were measured immediately after surgery and at 2, 6, and 12 months postoperatively. At 12 months, patients were assessed using the Patient and Observer Scar Assessment Scale.

Results The lesion locations included the face, arms, legs, back, and abdomen. The anchoring method resulted in shorter and smaller scars than the conventional method. There were no cases of postoperative hematoma or wound dehiscence. Significant differences in postoperative scar widening were found in the arm and leg areas $(P<0.05)$.

Conclusions The anchoring method introduced in this study can provide much better outcomes than the conventional method. The anchoring method is particularly useful for the removal of CMN around the joints or extremities, where the surgical site is subjected to high tension.
\end{abstract}

Keywords Nevus / Scar / Superficial fascia
Correspondence: Hook Sun Department of Plastic and Reconstructive Surgery, Busan Paik Hospital, Inje University School of Medicine, 75 Bokji-ro, Busanjin-gu, Busan 47392, Korea

Tel: +82-51-890-6136 Fax: +82-51-894-7976 E-mail: sun443@naver.com

Received: March 19, $2020 \bullet$ Revised: October 8, $2020 \bullet$ Accepted: October 16, 2020

pISSN: 2234-6163 • elSSN: 2234-6171 • https://doi.org/10.5999/aps.2020.00458 • Arch Plast Surg 2021;48:55-60

\section{INTRODUCTION}

Congenital melanocytic nevus (CMN) occurs in about $1 \%$ of newborns and should be removed to prevent it from turning into a malignant lesion [1]. The size of $\mathrm{CMN}$ varies from $1 \mathrm{~cm}$ to over $20 \mathrm{~cm}$. The conventional surgical method is elliptical excision. However, with this method, it may be difficult to remove a large lesion at once; therefore, staged excision may be required in such cases [2]. In addition, scar widening may result from the high tension created by the excision and repair of a large area of 
tissue. Various surgical methods have been studied to solve these problems [3]. The number of surgical procedures can be reduced if the first postoperative scar is relatively short and narrow, which would also reduce the financial burden faced by patients and their families. In light of these issues, in this study, we introduce a method to effectively excise lesions while minimizing scarring and compare its outcomes to those of existing surgical methods.

\section{METHODS}

We collected and compared data on patients who underwent surgery using the anchoring technique $(n=42)$ and those who underwent surgery using the conventional elliptical technique $(n=36)$ between February 2016 and February 2019. Comparisons were made with respect to the patients' mean age, followup duration, and lesion location. Preoperative and postoperative photographs were taken to determine the lesion size and postoperative scar size immediately after surgery and at 2, 6, and 12 months postoperatively.

\section{Surgical technique}

An incision line was traced to divide the lesion through the center along its long axis (Fig. 1A). Based on this line, one side of the lesion was removed via en bloc resection up to the superficial fascia, including the dermis and fat, using a \#15 blade and an electrocoagulator (Bovie). The other side of the lesion, inclusive of the nevus-invaded dermal region, was removed via de-epithelialization using a \#15 blade (Figs. 1B and 2). The lateral margin of the lesion on its en bloc excision surface was then partially dissected to separate it partially from the underlying superficial fascia. The de-epithelialized side was also dissected from the top of the subcutaneous layer to form a dermal flap. The medial margin of the de-epithelialized dermal flap was then fixed by suturing it to the superficial fascia on the opposite side. A dog ear deformity was minimized by adjusting the anchoring position of the dermal flap to the fascia at both ends, or if already present, by pulling the dermal flap further to the opposite side for an-

\section{Fig. 2. Intraoperative photograph}

One-half of the nevus was removed via de-epithelization and the other half was removed en bloc, as in Fig. 1B.

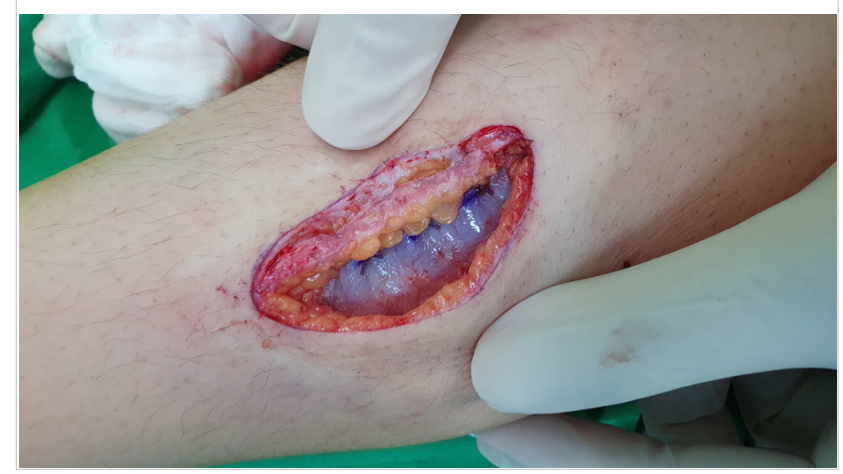

\section{Fig. 1. An illustration of surgical methods}

(A) An incision line was traced that separated one-half of the nevus from the other half along its long axis. (B) One-half of the nevus was removed via de-epithelization, including a portion of its dermis. The other half was removed en bloc, from its subcutaneous layer up to the superficial fascia. (C) Dissection of the dermis of the de-epithelialized part, creating a dermal flap, fixed on the opposing superficial fascia. The fixation position was adjusted to minimize the redundant skin. (D) Finally, the wound was doubly secured through the dermis to the dermis suture, thereby creating a strong holding force.
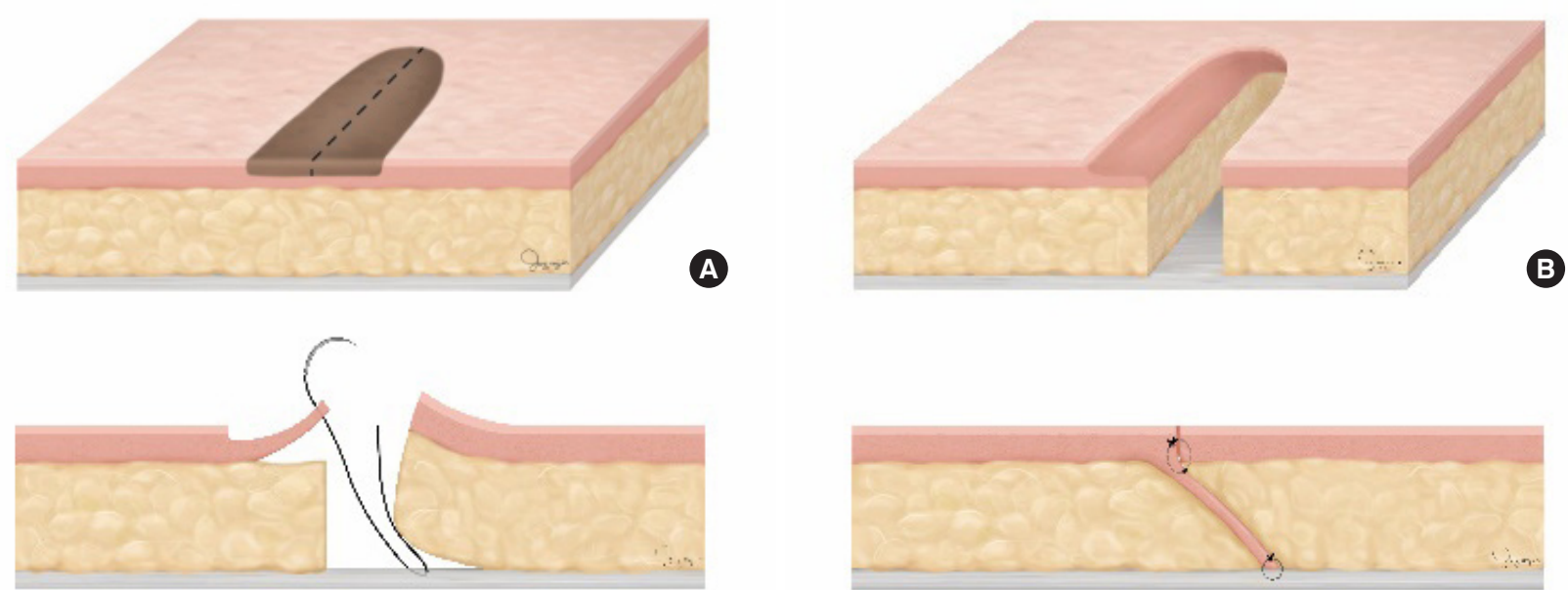

B

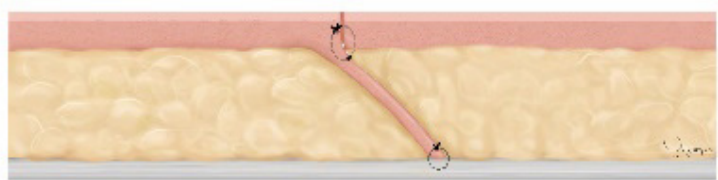


choring (Fig. 1C). The height of the dermis at both ends was then matched by trimming the redundant fat. Finally, the dermal margins from both sides were closed (Fig. 1D), and the skin edges were sutured together.

\section{Assessment}

The length of the lesion's long axis and amount of scar widening were measured immediately after surgery and at 2, 6, and 12 months postoperatively (Fig. 3). Patients were assessed at 12 months postoperatively using the Patient and Observer Scar Assessment Scale (POSAS), which was designed to evaluate various types of scars subjectively. Statistical analysis was performed using SPSS version 12.0 (SPSS Inc., Chicago, IL, USA). Operative outcomes were compared using the independent $t$-test, and P-values less than 0.05 were considered to indicate statistical significance.

\section{Fig. 3. Scar analysis method}

Length of the long axis of the scar (red line), and width of the scar (yellow line).

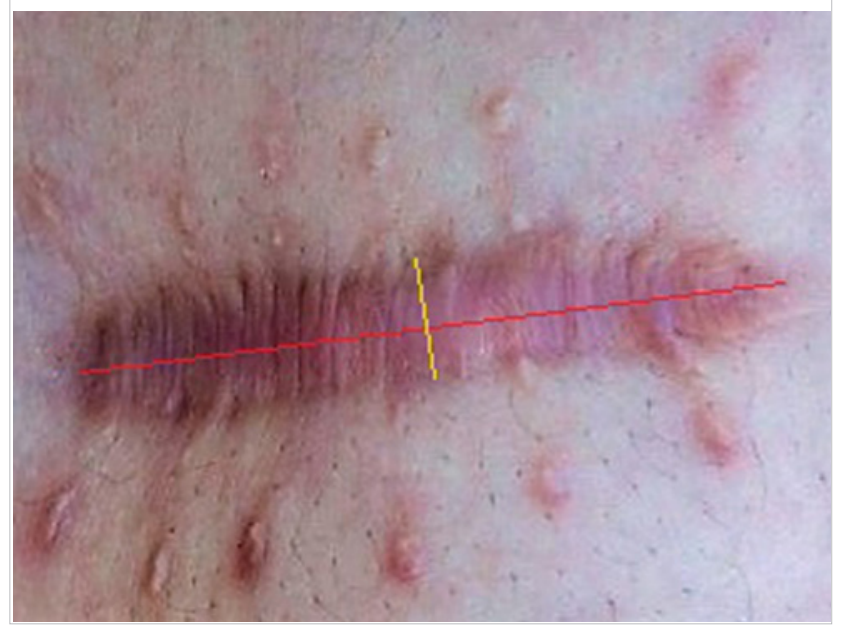

Table 1. Differences between the calculated and measured two-dimensional extent of the defects

\begin{tabular}{|c|c|c|c|c|}
\hline \multirow{2}{*}{ Site } & \multicolumn{2}{|c|}{$\begin{array}{l}\text { Conventional method } \\
\qquad(n=42)\end{array}$} & \multicolumn{2}{|c|}{$\begin{array}{l}\text { Anchoring method } \\
\qquad(n=36)\end{array}$} \\
\hline & $\begin{array}{l}\text { Length } \\
\text { (cm) }\end{array}$ & $\begin{array}{l}\text { Width } \\
\text { (cm) }\end{array}$ & $\begin{array}{l}\text { Length } \\
(\mathrm{cm})\end{array}$ & $\begin{array}{l}\text { Width } \\
(\mathrm{cm})\end{array}$ \\
\hline Face & 2.1 & 0.9 & 1.7 & 1.4 \\
\hline Arm & 5.7 & 3.5 & 7.1 & 4.5 \\
\hline Leg & 17.5 & 10.4 & 8.7 & 6.1 \\
\hline Back & 8.2 & 4.4 & 15.1 & 9.4 \\
\hline Abdomen & 7.4 & 5.2 & 9.7 & 6.3 \\
\hline
\end{tabular}

Values are presented as mean.

The preoperative congenital melanocytic nevus size was the longest in the leg and smallest in the face in the conventional group.

\section{RESULTS}

The sizes of the CMN varied widely, from 7 to $300 \mathrm{~mm}$ (mean, $72 \mathrm{~mm}$ ) (Table 1). The size of CMN did not show a significant difference between both groups $(\mathrm{P}<0.05)$.

Both the anchoring and conventional methods resulted in postoperative lengthening of the scar's long axis as well as widening; however, the anchoring method showed a smaller increase in both lengthening and widening than the conventional method. The lesion locations included the face, arms, legs, back, and abdomen. The face was the most common location of $\mathrm{CMN}$, with 25 cases in the anchoring method group and 11 in the conventional method group (Table 2).

The greatest change in scar length was found for the abdomen and the most scar widening was observed for $\mathrm{CMN}$ of the leg. A significant difference in postoperative scar widening was found between the arm and leg areas $(\mathrm{P}<0.05)$. Changes in scars according to the method used (anchoring or conventional) were compared at 2, 6, and 12 months after surgery (Table 3, Fig. 4). In patients who underwent surgery using the conventional method, rapid scar widening took place in the arm after 6 months. In patients who underwent surgery using the anchoring method, rapid scar widening occurred after 6 months in the face, arm, and leg.

Three cases required dog ear repair surgery using the anchoring method. There were no cases of postoperative hematoma or wound dehiscence. The mean POSAS score was 3.6 \pm 0.9 , which was a satisfactory outcome (Figs. 5, 6).

\section{DISCUSSION}

CMN occurs in about $1 \%$ of newborns and should be removed because of its malignant potential. The main difficulty in excising $\mathrm{CMN}$ directly is related to its size and location. The larger

Table 2. Demographic characteristics of the anchoring group versus the conventional surgical method group

\begin{tabular}{lccc}
\hline Characteristic & $\begin{array}{c}\text { Anchoring } \\
\text { method }(\mathrm{n}=42)\end{array}$ & $\begin{array}{c}\text { Conventional } \\
\text { method }(\mathrm{n}=\mathbf{3 6})\end{array}$ & P-value \\
\hline Age (yr) & $14.2 \pm 3.7$ & $17.6 \pm 4.6$ & 0.252 \\
Follow-up period (mon) & $12.2 \pm 2.4$ & $14.8 \pm 4.7$ & 0.117 \\
Site & & & \\
$\quad$ Face & 25 & 11 & 0.359 \\
Arm & 4 & 7 & 0.094 \\
Leg & 2 & 8 & 0.165 \\
Back & 7 & 6 & 0.142 \\
Abdomen & 4 & 4 & 0.297 \\
\hline
\end{tabular}

Values are presented as mean $\pm S D$ or number.

There was no significant difference between the two groups in terms of patients' demographic characteristics. 
Table 3. Postoperative scar analysis

\begin{tabular}{|c|c|c|c|c|c|c|}
\hline \multirow[t]{2}{*}{ Site } & \multicolumn{2}{|c|}{$\begin{array}{l}\text { Average difference in the length of the long } \\
\text { axis pre- and postoperativelya) }\end{array}$} & \multirow[t]{2}{*}{ P-value } & \multicolumn{2}{|c|}{$\begin{array}{l}\text { Average amount of postoperative scar } \\
\text { widening }\end{array}$} & \multirow[t]{2}{*}{ P-value } \\
\hline & Anchoring method & Conventional method & & Anchoring method & Conventional method & \\
\hline Face & $4 \pm 1.2$ & $5 \pm 1.0$ & 0.255 & $1 \pm 0.7$ & $3 \pm 0.5$ & 0.178 \\
\hline Arm & $15 \pm 2.7$ & $18 \pm 4.4$ & 0.110 & $4 \pm 1.2$ & $7 \pm 2.4$ & $0.021^{c)}$ \\
\hline Leg & $22 \pm 5.1$ & $30 \pm 7.2$ & 0.336 & $8 \pm 3.5$ & $15 \pm 2.8$ & $0.047^{c}$ \\
\hline Back & $27 \pm 6.7$ & $38 \pm 5.5$ & 0.412 & $5 \pm 3.7$ & $11 \pm 3.1$ & 0.210 \\
\hline Abdomen & $25 \pm 8.2$ & $39 \pm 7.4$ & 0.247 & $4 \pm 1.2$ & $7 \pm 2.6$ & 0.375 \\
\hline
\end{tabular}

Values are presented as mean \pm SD.

a)Average length differences of the long axis preoperatively and postoperatively (length of the scar's long axis at 6 months postoperatively) - (preoperative length of the

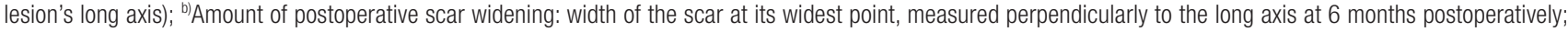

c) P-values $<0.05$ indicate statistical significance.

\section{Fig. 4. Differences in scar widening by time}

(A) Differences in scar widening in the face. (B) Differences in scar widening in the arm. (C) Differences in scar widening in the leg. (D) Differences in scar widening in the back. (E) Differences in scar widening in the abdomen.
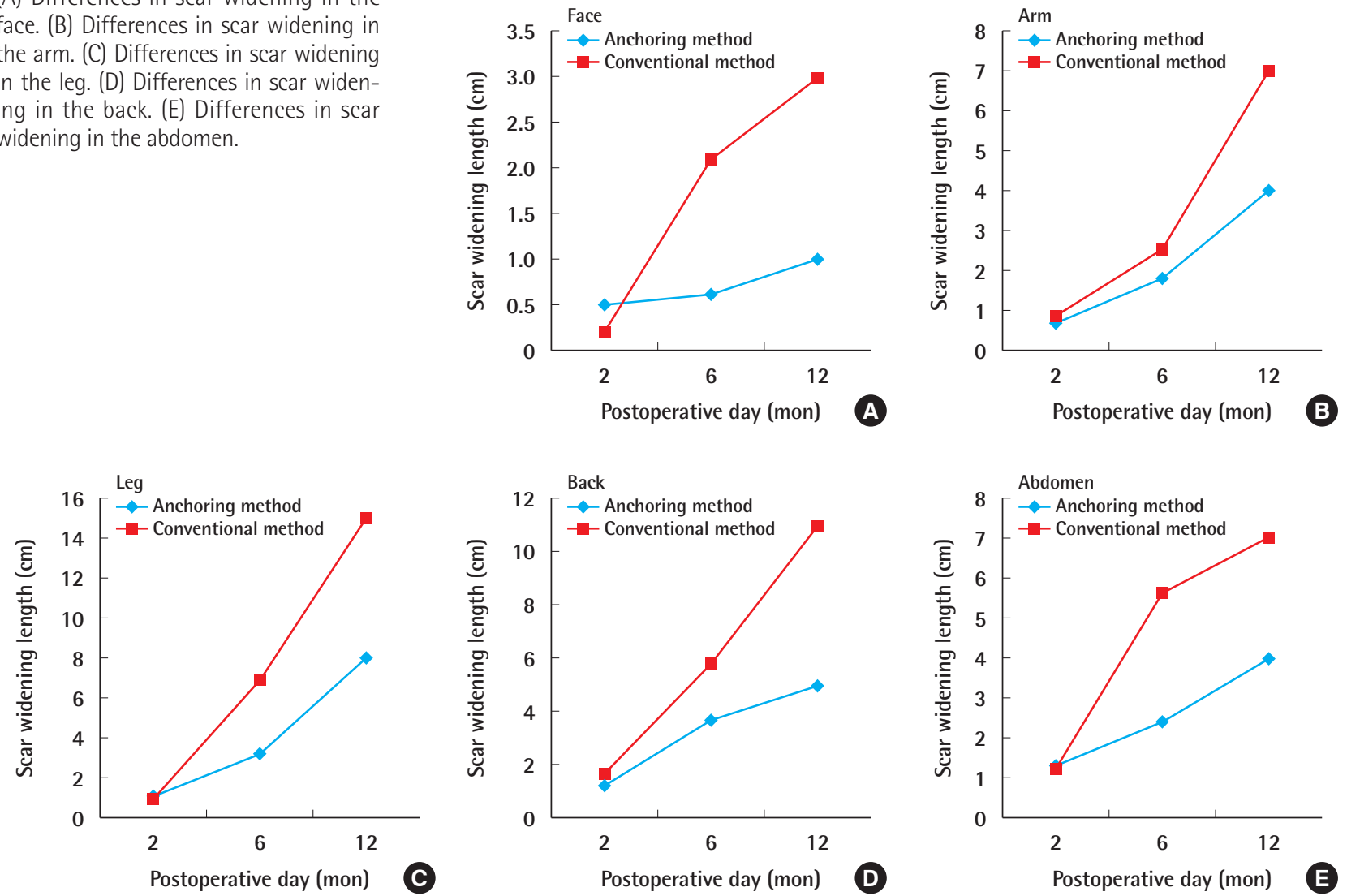

the lesion, the more difficult it is to remove at once; additionally, if excessive measures are taken to remove a large lesion, the scar becomes wider as the tension on the wound increases $[4,5]$. In particular, lesions on the face, which is a major location of many cosmetically important structures, can lead to distortions due to pulling [6]. Another point to consider is the shape of the lesion, as CMN can present with various shapes, including linear, round, and irregular. After excision of irregular lesions, dog ear deformities can develop during skin closure, and healthy skin must be excised to correct them.

In the conventional surgical method, elliptical excision performed to minimize the risk of dog ear formation creates a scar that is longer than the lesion along the long axis. Because a length-to-width ratio of 3-4:1 is required in order to prevent dog ear formation, the scar length must be increased as the shape of the lesion becomes rounder. 


\section{Fig. 5. CMN located in the right temple}

(A) Preoperative photograph $(50 \times 15 \mathrm{~mm})$. (B) Postoperative photograph at month 6, showing a 54-mm linear scar with 2-mm scar widening, no dog ear deformity, and no distortions of the periorbital structure. CMN, congenital melanocytic nevus.
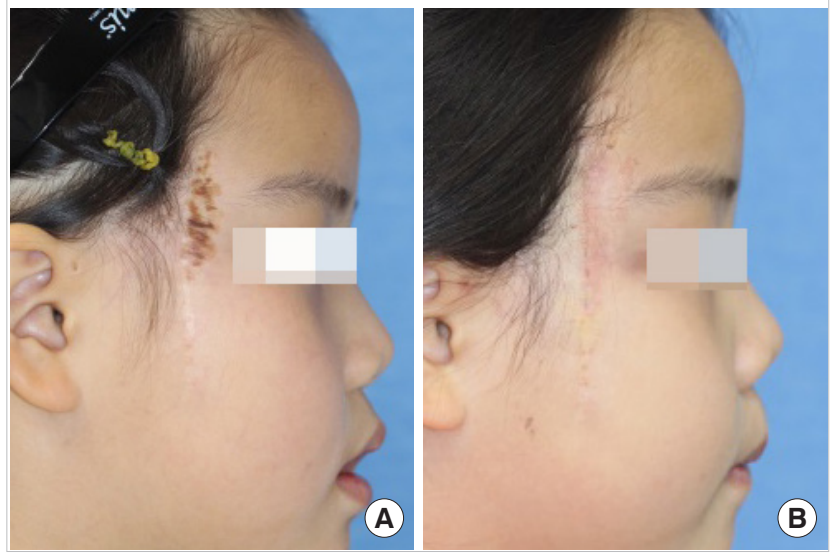

The anchoring method, in contrast, requires an excision design that is larger than the lesion. The dermal flap could be fixed to a different location on the fascia depending on the shape of the lesion. The rounder lesion shape results in a higher risk of dog ear formation; however, when the dermal flap is pulled closer to the center of the lesion for fixation to the fascia, a smaller dog ear is produced because of retraction of the redundant skin. The resultant scar length is shorter than that obtained with the conventional method.

In the conventional method of closing a defect after lesion excision, the opposite sides of the wound are aligned vertically. However, in the anchoring method, the de-epithelialized dermis descends diagonally and is anchored to the superficial fascia, thereby increasing the contact area between tissues. As a result, this creates less scar widening during the healing process, even when the tissue is under tension. Because the dermal flap is fixed to the fascia and the fixation in the upper layer is intradermal, further scar widening is presented. Our postoperative scar data revealed that leg lesions showed the greatest change in scar widening. In areas with more movement, the wound was placed under greater tension; therefore, scar widening was more likely to occur [7]. A significant difference in postoperative scar widening was found in the arm and leg area $(P<0.05)$, meaning that better results can be obtained by using the anchoring method near the joints or in the extremities where there is more motion. However, according to our findings, the anchoring method can be used in all areas of the body, regardless of the lesion location, to reduce the scar length and width.

A major limitation of this study is the size of our patient population, as more statistically accurate results could have been obtained with a greater number of cases. It was also difficult to fully

\section{Fig. 6. CMN located on the right buttock}

(A) Preoperative photograph $(147 \times 112 \mathrm{~mm})$. (B) Postoperative photograph at month 6 , showing slight scar widening $(162 \times 8$ $\mathrm{mm}$ ), and dog ear deformity occurring postoperatively (first stage, at the sixth postoperative month). The scar and dog ear can be eliminated via a second-stage surgical procedure. CMN, congenital melanocytic nevus.
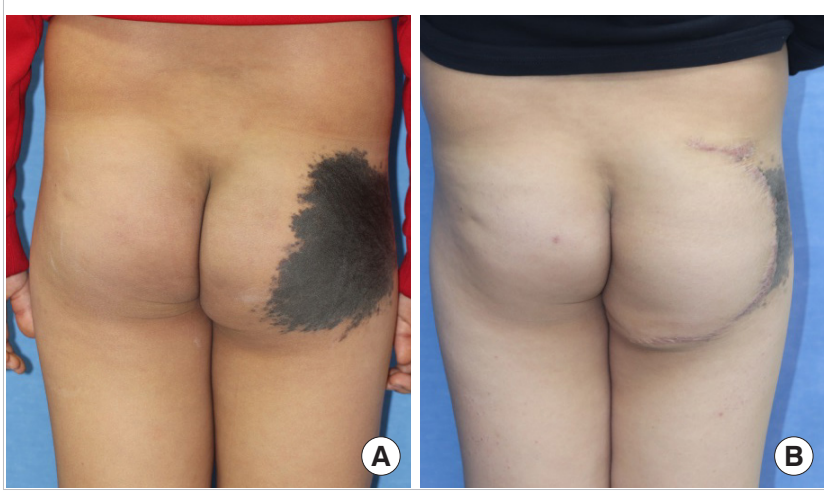

control for factors that could affect the scars, such as postoperative surgical site movement and scar management.

The anchoring method introduced in this study can provide much better outcomes than the conventional method. The anchoring method may be particularly useful for the removal of $\mathrm{CMN}$ around the joints or in the extremities, where the surgical site is subjected to high tension. In addition to the removal of CMN, this method has the potential to be widely used for other purposes, such as mass removal and repair of skin defects.

\section{NOTES}

\section{Conflict of interest}

No potential conflict of interest relevant to this article was reported.

\section{Ethical approval}

The study was approved by the Institutional Review Board of Inje University Paik Hospital (IRB No. 20-0048) and performed in accordance with the principles of the Declaration of Helsinki. Written informed consent was obtained.

\section{Patient consent}

The patients provided written informed consent for the publication and the use of their images.

\section{Author contribution}

Conceptualization: H Sun. Data curation: JW Kim, JY Yun, EH Chung, MJ Oh. Visualization: JW Han. Writing - original draft: JW Han. Writing - review \& editing: all authors. 


\section{ORCID}

Jin Woo Han https://orcid.org/0000-0002-2672-9954

Hook Sun https://orcid.org/0000-0003-0104-2598

Jin Woo Kim https://orcid.org/0000-0002-2672-9954

Ji Young Yun https://orcid.org/0000-0001-8129-1417

Eui Han Chung

Min Jun Oh https://orcid.org/0000-0003-1475-6426

\section{REFERENCES}

1. Michel JL, Chalencon F, Gentil-Perret A, et al. Congenital pigmented nevus: prognosis and therapeutic possibilities. Arch Pediatr 1999;6:211-7.

2. Hassanein AH, Rogers GF, Greene AK. Management of challenging congenital melanocytic nevi: outcomes study of

serial excision.J Pediatr Surg 2015;50:613-6.

3. Arneja JS, Gosain AK. Giant congenital melanocytic nevi of the trunk and an algorithm for treatment. J Craniofac Surg 2005; 16:886-93.

4. Mutti LA, Mascarenhas MR, Paiva JM, et al. Giant congenital melanocytic nevi: 40 years of experience with the serial excision technique. An Bras Dermatol 2017;92:256-9.

5. Hudson-Peacock MJ, Matthews JN, Lawrence CM. Relation between size of skin excision, wound, and specimen. J Am Acad Dermatol 1995;32:1010-5.

6. Arneja JS, Gosain AK. Giant congenital melanocytic nevi. Plast Reconstr Surg 2007;120:26e-40e.

7. Bayat A, McGrouther DA, Ferguson MW. Skin scarring. BMJ 2003;326:88-92. 